Aus der chirurg. Abteilung des Diakonissenhauses zu Posen (leitender Arzt: Geh. Med.-Rat Prof. Dr. Borchard).

\title{
Akute progrediente Encephalitis, akute zirkumskripte Meningitis und Meningoencephalitis.
}

Von Prof. Dr. Borchard.

Die akuten Formen der Encephalitis, Meningoencephalitis und Meningitis bilden ein Gebiet, auf dem unsere operative Tätigkeit unter dem Gesichtspunkte am meisten eingesetzt hat und einsetzen wird, in Zunahme begriffene Hirndrucksymptome allgemeiner wie lokaler Natur aufzuhalten. Wenn wir uns vor Augen führen, da $B$ allgemeine eitrige Hirnhautentzündungen durch Operation geheilt sind ( $\mathrm{K}$ ü $\mathrm{m}$ m ell), so kann man sich diesen Heilungprozeß doch nur so vorstellen, daß durch die operative Druckentlastung des Gehirns günstige Momente für die Ausheilung des eitrigen Prozesses gegeben sind. Druck auf die Gefäße, Ödem wurde beseitigt, die Kompression lebenswichtiger Zentren behoben, die Resorptionsvorgänge begünstigt. Selbst noch so weitgehende Kraniektomien können den ganzen Herd nicht freilegen und wo sie bei zirkumskripterer Form dieser Aufgabe genügten, war es unmöglich, das infektiöse Material aus allen Maschen zu entleeren. Durch die Beseitigung des Druckes je vollkommener und nachhaltiger das erfolgt um so besser ... beheben wir vor allen Dingen die Momente, die das Gehirn selbst und seine Tätigkeit schwer schädigen und vernichten können, und geben den Vorgängen in den Gehirnhäuten Zeit auszuheilen. Daß hierbei die Kraniektomie wesentlich hilft, unterliegt keinem Zweifel. Gewiß kommen Spontanheilungen vor: gewiß sind auch durch Lumbalpunktionen Erfolge erzielt, man braucht aber gegenüber den augenblicklichen und augenscheinlichen Erfolgen der Operation nicht auf dem Standpunkte Briegers zu stehen, daß die bisherigen operativen Erfolge mehr Zufallserfolge sind. Wenn man auch den Krankheitsherd in 
seiner ganzen Ausdehnung nicht beseitigen und bloßlegen konnte, so war doch der günstige Ausgang der wohlverdiente Lohn einer überlegten, durch Druckentlastung günstig wirkensollenden Operation.

Anders, wesentlich erleichternd für die Indikationsstellung liegen die Verhältnisse, wenn neben oder ohne nennenswerte allgemeine Hirndrucksymptome akute Herderscheinungen einsetzen. Hier gibt uns die Umschriebenheit des Herdes die Hoffnung neben der Druckentlastung auch gleichzeitig den Krankheitsherd zu beseitigen. Zahlreich sind die Eingriffe noch nicht, welche unter diesen Gesichtspunkten vorgenommen sind, sie haben uns aber, wenn auch vereinzelt, schon alle in Betracht kommenden Krankheitsbilder vor Augen geführt: die akute Encephalitis, die Meningoencephalitis, die Meningitis circumscripta acuta. Alle drei Formen haben klinisch so viel Gemeinsames, daß es vorläufig noch überaus schwer wird, sie zu trennen, und es dürfte auch für die Zukunft keine allzu große Hoffnung auf Änderung bestehen; pathologisch-anatomisch sind die Prozesse wohl charakterisiert. Ein besonderer Fortschritt ist es, daß die letzten Jahre uns das Bestehen einer progredienten Encephalitis ohne Meningitis (Friedrich) gezeigt haben und gleichzeitig durch die Beobachtung Hahns, Friedrichs und Kochs erwiesen, daß diese entzündlichen Vorgänge im Gehirn operativ heilbar sind. Damit und mit den weiteren Beobachtungen der Meningitis serosa circumscripta wurden für die Chirurgen diese Erkrankungen in den Bereich ihrer Indikationsstellung gezogen.

Die Encephalitis haemorrhagica acuta, wie sie in der Rinde und der Marksubstanz oder in der Nähe des 4. Ventrikels als Folge von Intoxikationen und Infektionen auftritt und sich in dem Auftreten multipler Herde äußert, interessiert uns hier nicht. Uns soll nur die sich vornehmlich an einer Stelle, gewöhnlich nach Traumen lokalisierende Gehirnentzündung, beschäftigen, die durch ihre Tendenz zum Fortschreiten bedrohliche Erscheinungen hervorruft. Küttner sagt in seiner ausgezeichneten Abhandlung über Meningitis, ,daß die Ätiologie der traumatischen Meningitis ebenso wie der traumatischen Encephalitis in der Aktion bestimmter pathogener Mikroorganismen gegeben ist. Demgegenüber muß man daran festhalten, daß abakterielle, asep- 
tische, nicht toxische, sondern rein traumatische Encephalitiden vorkommen können, welche die Tendenz zum Fortschreiten haben. In der Natur der Sache aber liegt es und pathologisch-anatomisch ist es zwanglos zu erklären, daß die reaktiven entzündlichen Vorgänge in der Nähe eines Kontusionsherdes weiter die Gehirnmasse vernichten, vornehmlich unter dem Einfluß des begleitenden entzündlichen Ödems und ev. kleinerer Blutungen. Kommt eine Infektion zu einem Zertrümmerungsherde hinzu, so ist das Fortschreiten der Encephalitis durch die Zunahme der Entzündungserscheinungen ohne weiteres erklärt. Sind derartig infizierte encephalitische Herde immer die Vorläufer des Gehirnabszesses, wie Koch annimmt? Die Frage ist zu verneinen. Von der Virulenz der Bakterien, der Widerstandsfähigkeit des umgebenden Gẹewebes hängt es $a b$, ob der encephalitische Herd ausheilt oder nicht, zur zirkumskripten Eiterbildung (AbszeB) gelangt oder durch ganz rapiden Zerfall des Gehirns, Durchbruch in den Ventrikel vor der Abszeßbildung den Exitus herbeiführt. Es gibt aber eine Form der traumatischen Encephalitis, welche in ihrer Progredienz unter günstigen Bedingungen Halt machen kann, und es muß unsere Sorge sein, diese günstigen Verhältnisse zu schaffen und so lange zu erhalten, bis sich die Virulenz der Bakterien erschöpft oder die Widerstandsfähigkeit der Umgebung sowie die Regenerationsfähigkeit gekräftigt hat. Wohl kann die Lumbalpunktion durch Druckentlastung hier Erfolge erzielen, das souveräne Mittel aber ist die technisch gut ausgeführte Trepanation, die einmal eine längere Regelung des endokraniellen Druckes gewährleistet, dann uns auch die Möglichkeit bietet, den infizierten Hirnherd auszuschalten und zu drainieren. Vor allen Dingen muß berücksichtigt werden, daß unter Umständen durch Verlegungen oder Verwachsungen ein $A b f l u ß$ von Liquor bei Lumbalpunktion verhindert werden kann und daß gerade bei Encephalitis und Meningoencephalitis die Lumbalpunktion selbst bei größter Vorsicht nicht ganz ungefährlich ist.

Das Material der bisher operativ behandelten Encephalitiden ist nicht groß. Folgen wir der Darstellung Friedrichs, der sich um die Frage sehr große Verdienste erworben hat, so ist als ältester Fall der von Hahn 1895 operierte anzuführen, bei dem unter der Annahme eines raumbeengenden subkortikalen 
Prozesses nach einem Trauma die rechtseitige motorische Region freigelegt wurde. Es entleerten sich mehrere Kubikzentimeter grauroter, dünnbreiiger, erweichter Hirnmasse. A. v. Berg m a n $n$ fand bei der Sektion einer von der Treppe gestürzten 7ojährigen Person zwei Erweichungsherde im Gehirn, von denen der eine sich in den rechten Seitenventrikel erstreckte und glaubt, daß ein Eingriff rechterseits möglicherweise den letalen Ausgang und die weitergehende Erweichung der Hirnsubstanz aufgehalten hätte. Der von Friedrich mitgeteilte Fall bot sehr viel anamnestische Schwierigkeiten. Die Zunahme der Lähmung der linken Körperhälfte, die Benommenheit des Patienten zwangen aber 65 Stunden nach erlittener Verletzung trotz des Fehlens jeder nachweisbaren Fissur oder Depression, um die kleine äußere Schädelwunde als Zentrum einen Knochenlappen zu bilden. Unter demselben lagen flache Blutgerinnsel, ein das Krankheitsbild erklärendes epidurales Hämatom war aber nicht vorhanden. Die Dura zeigte ganz geringe Pulsation und in der Mitte einen anscheinend oberflächlichen Ril3, dem eine feine Fissur in dem Knochen des zurückgeschlagenen Lappens entsprach. Die Dura wurde gespalten. Zwischen Dura und Arachnoidea war ein ganz flacher geronnener Bluterguß, die Arachnoidea in ganzer Ausdehnung der freigelegten Teile blutig imbibiert, breiig veränderte, suffundierte, gelblich verfärbte Hirnsubstanz quoll langsam vor. Ein $3 \mathrm{~cm}$ langer Kanal führte in den Hauptherd der Erweichungszone. Die Operation hatte den Effekt, daß schon während derselben, die ohne Narkose ausgeführt wurde, das Bewußtsein wiederkehrte und der Patient beim Verbandwechsel den Hergang der Verletzung erzählen konnte. Er war durch einen Messerstich am Schädel verletzt worden, war selbst zu Bett gegangen, hatte in der Nacht mehrmals erbrochen und gemerkt, daß er den linken Arm und das linke Bein nicht bewegen konnte. Am Morgen konnte er den Kopf und Rumpf nicht mehr erheben. Die Operation brachte Heilung. In der Epikrise betont Friedrich, daß nach der Stichverletzung des motorischen Rindengebietes die ersten nach mehreren Stunden auftretenden Funktionsstörungen der linken Körperhälfte auf blutige Imbibition der motorischen Zentren zu beziehen seien, daß die nach einem Intervall von zwei Tagen aber einsetzende Apathie und Benommenheit beim Fehlen jedes Zeichens einer 
erneuten Blutung nur sich aus der Infektion des Zertrümmerungsherdes erklären ließe. Seine Annahme, es handle sich um eine progrediente infektiöse Encephalitis ohne Beteiligung der Meningen hatte sich bestätigt. Das prinzipiell Wichtige des Falles ist, daB das ganze Krankheitsbild entstanden war, ohne begleitende Gehirnhautentzündung, trotzdem der Verletzungsweg durch die Meningen ging und daß diese fortschreitende Entzündung und Erweichung durch die Operation aufgehalten und geheilt werden konnte. So wichtig das erste in rein wissenschaftlicher Beziehung ist, so wird es doch durch die praktische Bedeutung des zweiten in den Schatten gestellt. Mußten wir doch früher bei der Ähnlichkeit der encephalitischen und meningitischen Symptome Enttäuschungen in unserer Diagnose bei der Operation befürchten, die bei der erwiesenen operativen Heilbarkeit jedes der Prozesse jetzt für den Patienten bedeutungslos ist. Außer diesen Beobachtungen habe ich in der Literatur nur noch einen Fall von augenscheinlich aseptischer Encephalitis gefunden, der sechs Monate post trauma wegen stechender Kopfschmerzen die Trepanation sowie das Herauspräparieren der gelblichroten Gehirnmassen nötig machte (Brewitt), den Fall von Borseck und Wizel, bei dem durch das Trauma Aphasie, Hemiparese bedingt war und sechs Tage später Hemiplegie, Bewußtseinsverlust einsetzte. Durch Operation wurden zwei Teelöffel blutiger, mit Gerinnseln durchsetzter Flüssigkeit entleert. Wenn auch die Verfasser das Ganze als ein traumatisches Blutextravasat in die weiche Gehirnsubstanz deuten, so wird man doch wohl das Richtigere treffen mit der Annahme, daß es sich um eine traumatische Encephalitis mit reichlicherer Blutbeimischung gehandelt hat. Der Fall Kochs von akuter progredienter Encephalitis nach Trauma bietet an und für sich kein besonderes Interesse, da die fortschreitenden Gehirnentzündungen nach größeren komplizierten Schädelfrakturen so häufig, ihre Erklärung durch die Infektion so leicht, die Therapie in der sachgemäßen Behandlung der komplizierten Schädelfrakturen überhaupt gegeben ist. Auch der Durchbruch in den 'Ventrikel ist bei der of $\mathrm{f}$ en e $\mathrm{n}$ fortschreitenden Encephalitis ein Ereignis, das viel weniger Bedeutung hat als wie bei der geschlossenen Form der Gehirnentzündung.

Ebenso wie diese speziellen Mitteilungen haben uns alle Be- 
obachtungen, welche dies Gebiet streiften, die eingehenden Abhandlungen Oppenheims über Encephalitis erwiesen, daß es eine traumatische Form der Gehirnentzündung gibt, welche gewöhnlich infolge einer Infektion, zuweilen aber auch ohne eine solche, bei geschlossenen oder scheinbar geschlossenen Schädeln die Tendenz hat, weiter fortzuschreiten. Sie tritt nach zwei bis drei Tagen gewöhnlich unter Zunahme der allgemeinen Drucksymptome, der Benommenheit usw. und vor allen Dingen der lokalen Herdsymptome in die Erscheinung, ruft bald bedrohliche Zeichen hervor und ist operativ heilbar. Der Möglichkeit spontaner Ausheilung in leichteren Fällen habe ich bereits gedacht, ebenso wie der Möglichkeit des Überganges in einen Abszeß. Nur hinweisen möchte ich noch darauf, daß wir denselben Prozeß bei den offenen Schädelfrakturen und gleichzeitigen Gehirnverletzungen häufig vor unseren Augen haben verlaufen und heilen sehen. Die Heilungsmöglichkeit ist dann gegeben durch die komplizierte Fraktur, also die Lücken im Schädel, die den Ausgleich der Drucksteigerung, die Entleerung und Abstoßung der entzündeten Hirnmassen ermöglichte und eine Drainage zuließ. Denselben Prozeß innerhalb eines geschlossenen oder nicht genügend eröffneten Schädels bietet jenes von Friedrich beschriebene Krankheitsbild und wartet nur darauf, daß wir ihm rechtzeitig die Tore zur Heilung öffnen. Nur die akute progrediente Encephalitis nach Trauma bei geschlossenem oder scheinbar geschlossenem Schädel ist es, welche in der Reihe dieser Betrachtungen unser Interesse verdient und auch überhaupt besondere Berücksichtigung beansprucht.

Die Frage, ob nur die infizierten Encephalitiden die Neigung zur Progredienz haben, möchte ich hier nur streifen. Ich habe diese Frage in der deutschen Chirurgie ausführlicher erörtert und bin zu der Überzeugung gekommen, daß auch ohne primäre oder sekundäre Infektion eine traumatische Encephalitis die Tendenz zum weiteren Fortschreiten haben und deutlich durch äußere Erscheinungen markieren kann.

Nahe verwandt nicht nur in den klinischen Erscheinungen, sondern auch in der Pathogenese ist mit dieser Form der Encephalitis die Meningitis serosa acuta circumscripta. Zum Verständnis dieses Krankheitsbildes seien einige anatomische Bemerkungen 
am Platze. Nach Strobes Angaben besteht die Leptomeninx aus einer äußeren und inneren Grenzlamelle und einem zwischen beiden liegenden Lymphraum, durch welchen sich ein an beiden Lamellen aufgehängtes Maschensystem von Platten und Strängen ausspannt. Zu zirkumskripten Hydropsien im Arachnoidalraum kann es also nur kommen, wenn gewisse Stellen durch pathologische Adhäsionsbildung gegen den Hauptraum abgeschlossen werden. Solche Adhäsionen treten nach Traumen, entzündlichen Affektionen des Zentralnervensystems und seiner Häute ein. Diese Cystenbildung kann auch während des akuten Anfalls einsetzen und je nach ihrem Sitz zu Herderscheinungen führen. Das Großhirn ist häufiger beteiligt wie das Kleinhirn, wohl aus dem Grunde, weil an ersterer Stelle Traumen häufiger sind. Gewisse Gegenden sind anatomisch zur Cystenbildung prädisponiert, weil hier schon normalerweise Hohlräume existieren, so die Cisterna chiasmatis, interpeduncularis trigemini, acustico facialis, cerbello-medullaris. Wenn auch die Verbindung zwischen Ventrikeln und Subarachnoidalraum die Regel ist und somit die Meningitis externa und interna sehr häufig sich vergesellschaften, so ist doch bei einem Verschluß des For. Magendi diese Kommunikation gestört. Bei Leuten, welche ein Schädeltrauma erlitten oder eine Entzündung im Bereich der Gehirnhaut durchgemacht haben, können Störungen in der Füllung der Gehirngefäße den Anstoß zu stärkerer seröser Exsudation bieten. Die Bildung des Liquor ist als eigentlicher Sekretionsvorgang aufzufassen, der ebensosehr von dem Zustande der meningitischen Blutgefäße wie von dem der Randendothelien und von Nerveneinflüssen, welche beide beherrschen, abhängig ist. Die äußeren Meningen erkranken leichter, weil sie Traumen und Infektionen mehr ausgesetzt sind und weil sie bei ihrem größeren Arteriensystem eher in die Lage versetzt werden, infektiöses Material vom Blute her aufzunehmen. Nach der einfacheren Darstellung $\mathrm{Heules}$ stellt die Arachnoidea ein wassersüchtiges und gewöhnlich lockeres Bindegewebe dar, in welchem der Flüssigkeitsaustausch sehr rasch erfolgt. Bei Entzündungen kommt es in diesem nicht allein zur Adhäsionsbildung und vermehrter Exsudation, sondern auch die Resorption wird behindert. So klar und einfach diese Heulesche Darstellung auch ist, so warm auch gerade Oppenheim für sie eintritt, so dürfte sie 
doch nicht ganz allen Tatsachen gerecht werden, so daß die Darlegungen Ströbes, welche sich im allgemeinen mit denen $Q$ u inckes decken, mir annehmbarer erscheinen. Wie dem auch sei, jedenfalls stimmen darin alle überein, daß infolge bestimmter ätiologischer Momente sich Adhäsionsbildungen innerhalb der weichen Hirnhaut vollziehen, welche bei gleichzeitiger Änderung der Exsudation und Resorption zu vermehrter Flüssigkeitsansammlung (Cystenbildung) führen können. Auch Transsudationsvorgänge können bei dem Druck auf die kleineren und größeren Venen hierbei eine Rolle spielen.

Ätiologisch kommen eigentlich nur zwei Momente in Betracht : Irauma und Infektion. In vielen Fällen werden beide sich vereinigen. Der sehr interessante Fall $\mathrm{A} x \mathrm{~h}$ a u sens wird vom Verfasser als rein traumatisch gedeutet, ebenso der von Janz beschriebene. Als rein infektiöser Natur ist die Erkrankung im Falle Wendels aufzufassen, wo nach einer eitrigen Liderkrankung sich eine umschriebene Meningitis ausbildete. Nach dem Aussehen des Exsudates ist die Genese nur selten zu bestimmen, ebenso kann auch die bakteriologische Untersuchung der Cystenflüssigkeit oder des Lumbalpunktates im Stich lassen. Nach den experimentellen Untersuchungen Streits setzen die regressiven Prozesse schon am 5. Tage ein. Als Reste der akuten bakteriellen Meningitis können umschriebene Zellinfiltrate zurückbleiben. Eine anfangs eitrige umschriebene Meningitis kann in eine seröse übergehen. Wie die bei Otitiden häufig beobachteten umschriebenen Meningitiden beweisen, brauchen die in der Nähe eines Eiterherdes sich ausbildenden entzündlichen Flüssigkeitsansammlung nicht immer eitriger Natur zu sein. Fernerhin gibt es nach $\mathrm{Zabel}$ eine Meningitis purulenta aseptica. Remlinger fand in diesen Fällon, bei denen alle bakteriologischen Untersuchungsmethoden, auch Tierimpfung, negativ blieben, mikroskopisch polynukleäre Leukocyten mit vereinzelten mononukleären. Also weder die makroskopische, mikroskopische, noch bakteriologische Untersuchung kann uns in jedem Teile Aufschluß über die Genese geben, und nur, wenn wir Trauma oder die Infektionsquelle mit Bestimmtheit ausschließen können, werden wir uns uneingeschränkt für dieses oder jenes Moment entscheiden können. Auch toxische Einflüsse allgemeiner Natur muß man berücksichtigen. 
So berichten Benders und Nieuwenhuyse über einen Fall von Meningitis serosa circumschripta corticalis bei einer 76 jähr. Frau im AnschluB an eine akute Enteritis. Als anatomisches Substrat wurde eine Cyste mit serösem Inhalt gefunden, die mikroskopisch als Folge einer Leptomeningitis aufzufassen ist. Von Entzündung der Cortex war keine Rede. Bakterien wurden nicht gefunden. Auch ein großer Teil der nach Infektionskrankheiten einsetzenden Meningitiden ist eher einer Toxin- als direkten Bakterienwirkung zuzuschreiben. Chronische Infektionskrankheiten, wie Lues, Tuberkulose können ebenfalls zur Bildung einer zirkumskripten Flüssigkeitsansammlung in den Gehirnhäuten führen, wie z. B. Küttner eine umschriebene tuberkulöse Meningitis der Großhirnrinde beschrieben hat. Als anatomisches Substrat findet man bei der akuten umschriebenen Meningitis die Dura gespannt und injiziert, ihre Innenfläche feucht und glänzend. Die Arachnoidea kann leichte Trübungen aufweisen, ist meistens feucht, glänzend, durchsichtig. Das Exsudat ist klar oder leicht getrübt, von flüssiger oder mehr sulziger Konsistenz, füllt entweder nur die Sulci aus oder bedeckt die Gyri und kann selbst über dieselbe hervorragen. Der Übergang in die Umgebung ist zuweilen nicht scharf abgegrenzt. Besonders in frischen Teilen zeigen sich alle Übergänge des entzündlichen Prozesses in die Umgebung und auch das Exsudat flacht sich nach den Rändern ab. In den chronischen Fällen wiegt der umschriebene, deutlich sich abgrenzende Charakter vor. Schließlich existieren Fälle, in denen makroskopisch nichts Pathologisches sich zeigt und höchstens mikroskopisch sich Rundzelleninfiltration findet. Die Reichardtsche Hirnschwellung dürfte hierher zu rechnen sein.

Wie schon früher hervorgehoben, beweist das makroskopische Aussehen des Exsudates zuweilen nichts für den infektiösen Charakter. Wir werden in den akuten Formen vielmehr eine mehr oder minder ausgesprochene Trübung der Flüssigkeit finden, die unter Umständen einer Eiterbildung außerordentlich ähnlich sehen kann und die trotzdem nach und nach rein serös wird. Daher ist bei den chronischen Formen, besser aber gesagt in den Fällen, die erst später zur Operation oder Autopsie kommen, der seröse Charakter bei weitem vorherrschend. Durch die Form der Verwachsung innerhalb der Gehirnhaut wird die Gestaltung des 
Exsudates bedingt. Wohl das interessanteste Beispiel hierfür ist die Beschreibung Axhausens, der bei der Incision der Dura kristallklaren, unter höchsten Druck stehenden Liquor ausfließen sah. Rasch legt sich danach die hochgradig ödematöse Pia in Form großer, zartwandiger, wie Gallerte aussehende Blasen in den Spalt hinein. Stach man eine solche Blase an, so kam wieder etwas Liquor. Auch handbreit nach hinten bot sich dasselbe Bild. Es handelt sich also um eine exquisite Maschenbildung innerhalb des bullösen Ödems; bei Wendel war ein ähnlicher Befund. Inwieweit Trauma und Infektion auf das Aussehen des meningitischen Prozesses Einfluß haben, läßt sich vorläufig nicht entscheiden. Es dürfte sich aber empfehlen, für die Zukunft darauf zu achten, ob nicht bei Traumen anfangs und später die deutlich umschriebenere Form, bei Infektionen mehr die allmählich in normale Umgebung übergehende Form häufiger ist.

Bei den nahen Beziehungen der Häute zu der Gehirnsubstanz ist es eigentlich selbstverständlich, daß die letztere an dem Prozeß beteiligt ist. Da so häufig der Infektionsweg durch die Gehirnhaut erfolgt, auch bei Friedrich ist dies der Fall, so wird auch hier eine Reaktion, eine Beteiligung, wenn auch nur in den allerersten Stadien, nicht ganz gefehlt haben. Je nach' dem Vorwiegen der pathologischen Veränderungen an dieser oder jener Stelle müssen wir von Encephalitis oder Meningitis sprechen. Nun ist es aber auffallend, daß in der Literatur gerade bei den chronischen Formen der Meningitis serosa so häufig die absolute Intaktheit des Gehirns betont wird (Benders), während im akuten Stadium ausdrücklich die Mitbeteiligung der Rinde am Entzündungsprozesse hervorgehoben wird (Muskens). Krebs fand in seinem als Meningitis beschriebenen Falle starke Gefäßinjektion, Schwellung der Hirnsubstanz, Verfärbung der Rinde, also vielmehr Erscheinungen der Encephalitis wie der Meningitis. Die tatsächlichen Verhältnisse liegen demnach so, daß wir anfäng. lich die Kombination wohl in der weitaus größten Mehrzahl wenn nicht immer finden, da anatomisch ein entzündlicher Vorgang in den Hirnhäuten ohne wenn auch noch so geringe entzündliche Schwellung, kollaterales Ödem, Injektion der Gehirnrinde nicht denkbar ist. Erst wenn mit dem Abklingen der akuten Erscheinungen in den Gehirnhäuten oder umgekehrt die regressiven Ver- 
änderungen einsetzen, der Herd sich abkapselt, erst dann werden sich die sekundären Erscheinungen zurückbilden. In den Meningen werden sie aber durch die restierenden Verdickungen, Verwachsungen, Trübungen viel länger kenntlich bleiben und außerdem liegen sie dem Auge viel zugänglicher. Würden makroskopische oder mikroskopische Untersuchungen der angrenzenden Rindenteile bei der chronischen Meningitis circumscripta gemacht, so würde wohl bald der Beweis erbracht sein, daß der Prozeß anfangs mit einer Beteiligung der Hirnsubstanz verlaufen ist, selbst wenn bei der doch immer nur flüchtigen Untersuchung während der Operation sich Anhaltspunkte dafür nicht ergeben. $\mathrm{Da}$ im Interesse des Patienten aber kein Grund für die Excision eines Gehirnstückes vorliegt, so wird diese fast rein wissenschaftliche Frage wohl nur durch eingehendste Untersuchung bei Autopsien zu entscheiden sein.

Wir dürfen uns vorgreifend auf die Annahme stützen, daß in dem a kuten Stadium der zirkumskripten Meningitis immer eine Encephalitis der Rinde mit vorliegt - also eine Meningoencephalitis. Nur diese Tatsache erklärt uns das frühzeitige progrediente Auftreten von Herderscheinungen auch in den Fällen, in denen die entzündlichen Erscheinungen, insbesondere die Exsudatbildung in den Meningen so geringfügig sind und nur aus diesem Gesichtspunkte sind die schweren klinischen Symptome bei der venösen Hyperämie Gussenbauers, der Reichardtschen Hirnschwellung verständlich. In diagnostischer Hinsicht weist es uns aber auf die Unmöglichkeit hin, anfangs beide Prozesse auseinanderzuhalten, zumal die encephalitischen Prozesse, die chirurgisch uns interessieren, wohl ausschließlich der Rinde angehören.

Es würde zuviel Bekanntes wiederholen, wenn ich an dieser Stelle auf den klinischen Symptomenkompelx und die Diagnose der zirkumskripten Meningitis, Meningoencephalitis acuta in allen Einzelheiten einginge. Ich werde nur Einzelnes besonders Interessierendes herausgreifen. Da sind vor allen Dingen die Herderscheinungen. Pathologisch-anatomisch durch den Druck der zunehmenden Flüssigkeitsansammlung, der Gefäßfüllung in den Meningen, des kollateralen Ödems, der Entzündung in der Hirnrinde erklärlich, haben sie dementsprechend auch klinisch ihre Eigen- 
heiten. Sie treten nicht unmittelbar nach der Verletzung wie bei Gehirnzertrümmerung auf, sie setzen nicht so schnell wie bei primären traumatischen Blutungen ein, sondern sie entwickeln sich allmählich, und zwar wohl nicht vor dem zweiten Tage, bieten alle Übergänge vom Reiz bis zum schließlichen Lähmungsstadium, erfolgen entweder ohne vorherige, sich an das Trauma anschließende Gehirnsymptome oder summieren sich zu solchen. In der Anfangszeit neben den zunehmenden allgemeinen Drucksymptomen eine ausgesprochene Progredienz zeigend, können sie in jedem Stadium mit oder ohne therapeutische Maßnahmen Halt machen und sind einer völligen Rückbildung fähig, selbs t wenn sie auf infektiöser Basis beruhen. Die Progredienz ist nicht immer ein Zeichen der erfolgten In. fektion. Rein traumatische Meningitiden, Meningoencephalitiden gleichen in dieser Hinsicht den infektiösen Formen. Auch für die Encephalitis gilt dasselbe. Zuweilen gehen dem Auftreten der Herderscheinungen Anfälle kortikaler Epilepsie vorher oder kombinieren sich mit den ersten Erscheinungen. In dem schon erwähnten Falle Benders treten ohne äußerliche Ursache plötzliche Reizungserscheinungen des rechten motorischen Cortexgebietes in Form klonischer Krämpfe auf. Dieselben zeigten gewisse Eigentümlichkeiten, indem z. B. die Bewegungen nicht so schnell wie bei Epilepsie waren und die eine Hälfte der Bewegungen viel schneller als die andere erfolgte. In meinem unten zu erwähnenden Falle gingen zwei epileptische Anfälle zirka I 2 Stunden dem Auftreten typischer Herdsymptome voraus. Oppenheim beschreibt einen Fall von Schmerzhaftigkeit der linken Hinterhauptgegend bei Druck und Perkussion, Kopfhaltung nach links geneigt und nach rechts gedreht, Neigung zur Beugestellung nach links, Ausfallserscheinungen der rechten Körperseite, und betont, daß die Einwirkung dieser serösen Ergüsse auf das Gehirn sich durchaus nicht immer in ganz gleichmäßiger systematischer Weise geltend macht, daß vielmehr ein derartiges Überwiegen der Reiz- und Ausfallserscheinungen auf einer Seite bis zur Vorspiegelung einer Herderkrankung möglich ist.

Bei dieser Sachlage ist nicht die so häufige Andeutung, sondern das Vorhandensein ausgesprochener, vor unseren Augen sich entwickelnder Herdsymptome ein diagnostisches Cha- 
rakteristikum, das gleichzeitig unsere Indikationsstellung bestimmt.

Mit den Herdsymptomen können gleichzeitig die allgemeinen Hirnsymptome als Ausdruck des steigenden Druckes, der fortschreitenden Entzündung zunehmen. Zum Teil hängt das von der Größe des Traumas, der Schwere der Infektion ab, zum Teil aber auch von der Schnelligkeit, mit der sich die Entzündung in den Gehirnhäuten durch Verwachsungen abgrenzt. Auch diese allgemeinen Symptome treten erst ein oder erfahren eine Zunahme nach einer bestimmten Zeit (ca. 48 Stunden), sind entweder nur angedeutet oder steigern sich, wie im Falle Wendels, bis zum tiefen Coma, ohne dabei das Zeichen einer allgemeinen Gehirnhautentzündung sein zu brauchen.

Nicht so sehr durch ihr Vorhandensein wie durch ihre $\mathrm{Zu}$ nahme sind sie diagnostisch wie therapeutisch von Bedeutung.

Die Nackensteifigkeit ist als meningeales Reizsymptom in den akuten Fällen wohl stets vorhanden im Gegensatz zu der Seltenheit bei Tumoren, bei Blutungen, aber sie ist nur bei der Generalisierung der Entzündung in extremster Weise nachweisbar. Gewöhnlich ist sie angedeutet. Für den Übergang einer Meningitis externa in die interna spricht das plötzliche Auftreten eines aufwärts gerichteten Nystagmus als Ausdruck der Reizung der Vierhügelgegend ( $R$ üttlin). Die Prognose ist dann absolut schlecht.

Die Temperatur zeigt, wie bei allen akuten Gehirnerscheinungen, die verschiedensten Formen. Extrem hohe Steigerungen selbst im chronischen Stadium, nur ganz leichte Exacerbationen im akuten Anfang sind beobachtet. Wenn man bedenkt, von wie vielen Momenten gerade bei Gehirnerkrankungen dieses Symptom abhängig sein kann, wird man sich nicht wundern. Wichtig ist, daß die Abwesenheit von Temperatursteigerungen weder gegen eine Infektion noch gegen die Progredienz des Prozesses spricht. So ist es also in der Hauptsache das nach einem gewissen Zeitpunkte - ca. 48 Stunden - einsetzende Auftreten oder die Zunahme der allgemeinen Drucksymptome und der Herderscheinungen, das uns im Verein mit der anamnestisch festgelegten Tatsache eines Traumas, einer Infektion oder Intoxikation die Diagnose auf umschriebene akute Encephalitis, Meningitis, Meningoencephalitis stellen läßt und uns durch die Progre- 
dienz der Erscheinungen zum therapeutischen Handeln zwingt. Wir werden um so eher unsere Diagnose stellen, je näher der Prozeß einem Zentrum sitzt. Daß gerade in diesen Fällen unser therapeutisches Handeln erfolgreicher war, erklärt sich also einmal ungezwungen durch die frühere Diagnose, dann aber auch dadurch, daß gerade wegen der ausgesprochenen Herderscheinungen operativ der radikalere Weg, d.h. die Trepanation, gewählt wurde.

Wie kommt es, daß die Beobachtungen über die akuten Formen unserer Erkrankung in der Literatur so selten in Vergleich zu den ja auch nicht gerade häufigen chronischen sind? Daß die Mortalität ausgesprochener umschriebener akuter Meningitis, Meningoencephalitis eine so hohe und schnelle sein soll, entspricht erstens wohl nicht den tatsächlichen Verhältnissen, genügt aber auch zweitens nicht zur Erklärung. Wohl würde die Malignität, der foudroyante Verlauf die kleine Zahl der Operationen, aber nicht der Beobachtungen überhaupt verständlich machen. Der Grund liegt gerade auf der anderen Seite. Viele akute umschriebene Prozesse verlaufen so milde, daß sie irgendwelche Er. scheinungen anfangs nicht machen. Von den Encephalitiden nach Trauma ist dies lange bekannt, und Bergmann hat wiederholt darauf hingewiesen. Nach ihm brachte erst die Meningitis die Zeichen für die im Gehirn sich abspielenden entzündlichen Prozesse. Aber auch die milderen Formen der Gehirnhautentzündungen, und das sind gewöhnlich die umschrieben auftretenden, können ohne besonders alarmierende Symptome verlaufen und werden deshalb weniger beachtet. Aus ihnen aber kann sich die chronische Form entwickeln. Die spätere Zunahme des Prozesses beruht nicht auf der Weiterwirkung des entzündlichen und traumatischen Reizes, sondern, nachdem die Verwachsungen innerhalb der Meningen und mit dem Gehirn erfolgt sind, auf einer Änderung des Sekretions- und Resorptionsverhältnisses, wozu auch nervöse und lokale Blutfüllungszustände kommen können. Es kann selbst die anfangs harmloseste akute Form in der Folgezeit zu einer großen Meningitis serosa circumscripta chronica führen. Wenn auch in letzter Linie alles auf der Lokalisation und Umgrenzung des traumatischen oder sonstigen Reizes an einer bestimmten Stelle der Gehimoberfläche und der Meningen beruht 
und der Abkapselung dieses Herdes durch Verwachsungen, so sind doch die ursächlichen Momente für das Manifestwerden nach außen, für die Zunahme der Erscheinungen verschieden. Bei der akuten Form, Fortwirkung, Progredienz der Entzündung, sei es als Ausdruck der bakteriellen oder toxischen Virulenz, sei es des traumatischen Reizes oder der Kombination dieser Momente, bei der chronischen Form, Vermehrung und Ansammlung der Flüssigkeit, sei es in Form einer Cyste oder eines umschriebenen Ödems als Folge der durch die Verwachsungen bedingten Änderungen in der Zirkulation, Ausscheidung und Resorption des $\mathrm{Li}$ quor. In letzterer Beziehung können alle möglichen Momente von Bedeutung sein, nervöse Einflüsse, Insolationen, Intoxikationen, erneute Traumen. Eine chronische Encephalitis in diesem Sinne gibt es nicht. Liegt der Herd im Innern des Gehirns, ist er nicht zu groß, so füllt ihn Narbengewebe aus oder kapselt ihn so $\mathrm{ab}, \mathrm{da} \beta$ er für den Träger bedeutungslos ist. Die uns hier nur interessierenden Herde der Rinde werden bei nicht zu großem Umfang auch die Hirnhäute ausgefüllt und ausgekleidet und sind dann später in ihrem Aussehen und ihrem Verlauf gleichbedeutend mit den meningitischen Prozessen. Es sind also nur die schwereren Formen der akuten Prozesse, welche stark in die Augen springende Symptome machen und welche ein schnelles Handeln erfordern. Noch ist zwar keine Generalisation der Entzündung erfolgt, aber jeden Augenblick kann sie eintreten, noch sind Herdsymptome erkennbar, aber bald können sie in den allgemeinen Druckerscheinungen untergehen - noch ist es möglich, die weitergehende Encephalitis von dem Durchbruch in die Ventrikel, in lebenswichtige Zentren abzuhalten.

Die akuten, bis jetzt beschriebenen Formen stellen diese Grenzfälle dar, die durch einen glücklichen Zufall frühzeitig in Behandlung kamen, früh genug erkannt und operiert wurden. Ihre Zahl ist nicht groß, sie wird sich aber dadurch vermehren, $\mathrm{da} \mathrm{B}$ immer mehr auf sie geachtet wird, und vor der Generalisierung, vor der inoperablen Druckschädigung operiert wird. Ich bin fest überzeugt, daß auch Fälle in Zukunft operiert werden und auch jetzt schon operiert sind, die ohne Operation genesen wären. Was will das aber sagen bei der Sicherheit unserer Technik und 
der Unschädlichkeit der von einem Chirurgen ausgeführten Operation im Vergleich zu den Verlusten bei abwartenden Verfahren. Dieselben Bedenken haben wir bei allen Errungenschaften der Chirurgie durchgemacht. Es muß dem einzelnen Operateur überlassen bleiben, ob er - trotzdem noch nicht alle diagnostischen Momente erschöpft sind, trotzdem noch die Möglichkeit der Heilung ohne Operation besteht - schon aktiv vorgehen will oder nicht. Das „nil nocere" hat er aber mit der Operation mehr berücksichtigt, als ohne dieselbe, so paradox es auch klingen mag. Damit sind wir bei der Frage angelangt, haben sie eigentlich schon beantwortet, was wir therapeutisch tun sollen. $\mathrm{K} \ddot{\mathrm{u}} \mathrm{m}$ mell durch seine vorzüglichen Erfolge, Hinsberg, Brieger, Cohn, Lucae auf otologischem Gebiet haben uns gezeigt, daß selbst eitrige Meningitiden in jeder Form und jeder Ausdehnung heilbar sind, der Fall Friedrichs, die zahlreichen Beobachtungen von infizierter progredienter Encephalitis bei komplizierter Fraktur sprechen dafür, daß unter Umständen auch diese ausheilen können, wenn gewisse günstige Bedingungen erfüllt sind. Kein Gewebe ist so empfindlich gegen Zirkulationsstörungen wie das Gehirn. Alle jetzt in Rede stehenden Prozesse gehen aber mit Kreislaufstörungen, mit intrakraniellen Drucksteigerungen einher und es ist ein eigenartiger circulus vitiosus, daß die Entzündung, das Trauma durch Drucksteigerung die Zirkulation hindert und so den Ausgleich, die regressiven Vorgänge beeinträchtigt und daß bei künstlicher Minderung des Druckes die geschädigten Gewebe sich erholen und mit ihrer vitalen Kraft an Heilung und Abwehr beteiligen können. Gewiß haben wir bei universeller Meningitis es durch keine Methode in der Hand, den ganzen Krankheitsherd freizulegen und zu drainieren; nicht einmal bei akuter lokalisierter Meningitis ist es uns möglich, die Flüssigkeit völlig zu entleeren und genügend zu drainieren. Axhausen wie Wendel haben sich vergeblich darum bemüht. Gewiß werden wir bei progredienter Encephalitis nicht alle entzündete, infizierte Hirnmasse entferner. können, und wie schwer eine Drainage nach außen ist, weiß jeder, der es versucht hat. Man muß Brieger vollkommen beistimmen, daß die Heilung nicht auf eine bestimmte Form der Meningitis beschränkt ist, daß es auch nicht sicher ist, 
ob der Infektionsweg eine Bedeutung für die Heilbarkeit hat. Die Therapie schafft durch Ausschaltung des primären Eiterherdes und druckentlastende Maßnahmen nur Bedingungen, die den Eintritt der Heilung begünstigen. Man kann aber nicht die bisherigen operativen Erfolge als Zufallserfolge bezeichnen. Auch daß Spontanheilungen möglich sind, berechtigt noch nicht zu einem derartigen Standpunkt gegenüber den Operationen. Aus der Überlegung heraus, daß die endokraniellen Vorgänge bei dem Wachsen der allgemeinen und lokalen Symptome in Zunahme begriffen sind und Lebensgefahr oder dauernde Schädigung befürchten lassen, in der Überlegung, daß die starke Drucksteigerung die nächste und größte Gefahr darstellt und daß wir sie durch operative Maßnahmen beseitigen können, gestützt durch die Beobachtung, daß selbst schwer geschädigte Hirnregion nach der Druckregulierung sich erholen und bedrohte Partien geschützt werden können, müssen wir getreu der Vorschrift Kochers die Druckentlastung vornehmen, auch wenn wir nicht wissen oder feststellen konnten, um was es sich handelt. Das wird oft nur erst auf dem Operationstisch möglich sein; aber trotzdem ist dies angenehmer für den Arzt wie Patienten, als eine sichere Diagnose auf dem Obduktionstische. Wir wollen bis jetzt nur dieser vitalen Indikation genügen, müssen noch weiter die gefundenen Krankheitsbilder erklären, mit unserem Vorgehen vereinigen, und sind vorläufig noch weit davon entfernt zu sagen, es liegt diese Form der Meningitis, der Encephalitis vor, und deshalb muß diese oder jene Operation gemacht werden.

Zur allgemeinen Druckentlastung stehen uns zwei Mittel zur Verfügung, die Lumbalpunktion, die Trepanation. Erstere hat den Vorzug der schnellen und leichten Ausführbarkeit, der scheinbaren Ungefährlichkeit, letztere schafft eine dauernde Regulierung und kann Krankheitssitz, Krankheitsursache direkt angreifen und beseitigen. Beide haben ihre Nachteile. Da der Liquor sich leicht wieder ansammelt, muß die Lumbalpunktion öfter wiederholt werden, die Druckentlastung ist, da der Schädel geschlossen bleibt, gewöhnlich keine sehr erhebliche, Todesfälle scheinbar unmotivierter Art sind selbst nach Ablaufen weniger Kubikzentimeter beobachtet worden, so daß Oppenheim noch vor nicht langer Zeit vor der Anwendung warnte. Die Trepanation ist sicherlich der 
viel größere Eingriff. Ob es der schwerere für den Patienten 1st, möchte ich nicht so ohne weiteres zugeben, da bei schnellem und vorsichtigem Arbeiten die Freilegung der obersten Hirnpartien doch in kurzer Zeit vollendet ist.

Im speziellen stellen sich aber die Verhältnisse noch anders. Vielfach wurde die Lumbalpunktion aus diagnostischen Gründen unternommen. Sie wird uns über die allgemeine Zunahme des Liquor, über die Spannung, unter der er steht, über seine Beschaffenheit für gewöhnlich Auskunft geben, aber ein trüber Liquor bedeutet noch nicht immer eine eitrige Meningitis, die Erfolglosigkeit oder Geringfügigkeit der Punktion noch nicht immer das Fehlen der Drucksteigerung oder Flüssigkeitsvermehrung in der Schädelhöhle. Die Xanthochromie weist oft auf das anamnestisch nicht nachweisbare Trauma hin. Wir werden trotz einzelner Nachteile dieses wichtigen diagnostischen Hilfsmittels nicht entraten, wir werden etwaige momentane Besserungen infolge diagnostischer Lumbalpunktion gern mitnehmen, wir werden uns aber sagen müssen, daß die Lumbalpunktion zwar imstande sein kann, allgemeine Druckerscheinungen, die momentan bedrohlich sind, zu beseitigen, nicht aber gleichzeitig vorhandene progrediente Herderscheinungen aus der Welt zu schaffen. Haben allgemeine und lokale Erscheinungen keinen sehr hohen Grad erreicht, hält man aber eine Druckentlastung für gut, dann ist eine Lumbalpunktion am Platze. Sind die allgemeinen Symptome stärker, lebensbedrohlich, das Herdsymptom nur angedeutet - nicht progredient --, dann kann eine Lumbalpunktion versucht werden, um bei positivem Erfolge weiter abzuwarten ev. zu wiederholen. Wenn dagegen sich progrediente Herdsym. ptome ausbilden, immer deutlicher werden, die allgemeinen Druckerscheinungen nur angedeutet sind oder ganz fehlen, so soll an der Stelle des Herdes trepaniert werden. Konnten wir auch durch die Lumbalpunktion die allgemeinen Druckverhältnisse ändern, so ist diese Änderung doch in dem umschriebenen encephalitischen Herde, in der durch Verwachsungen abgeschlossenen Meningitis ohne Bedeutung. Bis dahin gelangt der Ausgleich nicht. Auch nach Parambolinis Ansicht sprechen Herdsymptome gegen die Lumbalpunktion.

Deshalb ist bei hohen allgemeinen Druckerscheinungen die 
Ergebnislosigkeit und Erfolglosigkeit der Lumbalpunktion, bei progredienter oder ausgesprochener Herderscheinung diese selbst die unbedingte Indikation zur Trepanation.

Die Erfahrungen der Otologen (Uffenorde) sind aus anderen Fällen gewonnen und somit deshalb etwas anders. Auch bei chronischer umschriebener Meningitis ist die Trepanation das einzig rationelle Verfahren. Nach Spaltung der Dura ist durch Ritzung der Arachnoidea die Flüssigkeit zu entleeren, durch Zerreißung der Stränge und Verwachsungen ein Rezidiv zu verhüten (Borchard). Das weitere Vorgehen richtet sich nach dem jeweiligen Befunde, der auch darüber entscheiden muß, ob man sich mit dem bis dahin Gefundenen zur Erklärung des Krankheitsbildes begnügen oder nach weiteren ev. encephalitischen Herden suchen will. Nachher dürfte es sich empfehlen zu drainieren bzw. tamponieren, jedenfalls aber durch Umkrempeln der Duralappen ev. Entfernung eines Knochenlappens für dauernde Druckregulierung zu sorgen.

Die bisher in der Literatur veröffentlichten Fälle von Meningitis serosa circumscripta a $\mathrm{cuta}$ sind nicht sehr häufig. Der von Axhausen mitgeteilte Fall ist wohl am frühsten zur Operation gekommen, weil bei ihm der Hirndruck schon am 6. Tage nach der Verletzung eine außerordentliche Höhe bei gleichzeitiger hoher Temperatur angenommen hatte. Da die Affektion das Stirnhirn betraf, waren keine Herderscheinungen vorhanden. $\mathrm{Ob}$ es sich, wie Axhausen meint, um eine rein traumatische Meningitis handelte, möchte ich dahingestellt sein lassen. Ich kann in dieser Beziehung den Ausführungen Axhausens nicht ganz beistimmen. An der Bedeutung der interessanten und wichtigen Mitteilung ändert aber das ätiologische Moment nichts. Der Fall Wendels reiht sich dem vorigen insofern an, als auch er das Stirnhirn betraf und wegen schwerer Hirndruckerscheinungen operiert werden mußte. Auch hier fehlte jede Herderscheinung. Wenn die infektiöse Liderkrankung 3 Monate vorhergegangen war, so ändert das nichts daran, auch diesen Fall seinem ganzen Verlauf nach als eine akute Meningitis aufzufassen.

Ch. Martin du Pa u sah bei einem Kinde mit Schädelfraktur vom 5. Tage an heftige Reizerscheinungen der Corticalis mit epileptiformen Anfällen und Benommenheit neben Parese des linken 
Armes und Facialis eintreten. Lumbalpunktion erfolglos. Nach der Duraspaltung entleerte sich aus der Arachnoidea reichlich Flüssigkeit.

Auch dieser Fall ist als eine akute Meningitis serosa anzusehen, bei dem neben allgemeinen Drucksymptomen ausgesprochene Herderscheinungen vorlagen. Wichtig ist, daß auch hier epileptische Anfälle auf die Reizung der Rinde hinwiesen, und ich glaube nicht fehlzugehen, wenn ich diesem Symptom bei der Differentialdiagnose zwischen Encephalitis und Meningoencephalitis acuta circumscripta eine größere Bedeutung beimesse.

In der Mitteilung Pitterleins handelt es sich um eine traumatische zirkumskripte Arachnitis adhaesiva, die durch einen Hufschlag gegen die linke Schläfe entstanden war, Erscheinungen von Rumpfmuskelschwäche, Schwindelerscheinungen (cerebelläre Erscheinung) bot. Bei der Trepanation in der Stirn- bzw. Schläfengegend entleerte sich viel Liquor, die weichen Hirnhäute waren glatt, glänzend gelblich verfärbt und wie mit dichtem Hauch bedeckt, ihre Fläche muldenförmig eingedrückt, trat nach Entleerung des Liquor in das Niveau zurück.

Diesen Mitteilungen schließt sich der von mir beobachtete Fall an.

A. S., Arbeiter, 35 Jahre alt, aufgenommen 13. VI. I9I3, entlassen 6. X. I9I3, erhielt vor drei Wochen bei einem Streite einen Schlag über den Kopf, wodurch eine offene Wunde entstand. Dieselbe heilte angeblich ganz gut, seit zwei Tagen aber stellten sich leichte Lähmungserscheinungen im linken Arm und Bein und Muskelzuckungen ein, nachdem I4 Tage nach der Verletzung reißende Kopfschmerzen in der rechten Kopfseite aufgetreten waren. Am gestrigen Tage soll ein Krampfanfall gewesen sein und die Lähmungserscheinungen wie Benommenheit zugenommen haben.

A uf nahmebefund: Mittelgroßer, kaum mittelkräftiger Mann, unterernährt. Herz, Lungen, Bauch ohne Besonderheiten. Pupillen mittelweit, reagieren gut. Okulomotorius ohne Besonderheiten. Linker Facialis leicht paretisch. Bewegungen im linken Arm und Bein werden kaum ausgeführt. Keine Parästhesien. Patellarreflexe beiderseits gleich gut. Sensibilitäts- und vasomotorische Störungen nicht nachweisbar. Bewußtsein deutlich getrübt.

Auf der rechten Kopfseite, an der vorderen Grenze des Scheitellappens, 2 bis $21 / 2$ Finger breit über dem Ohre eine eitrig belegte, granulierende Wunde, in deren Tiefe eine Verletzung des Knochens nicht vorhanden ist. Die Wundränder, die Umgebung der Wunde nicht ent- 
zündet. Temperatur 37,2 Grad morgens. Puls gespannt, 60 bis 70 Schläge.

Diag nose: Fortschreitende Encephalitis

Indikation. Trepanation.

Bei der sofortigen Operation wurde ohne Rücksicht auf die Weichteilwunde ein Hautknochenlappen über der rechten motorischen Rindenregion gebildet und mit elektrischer Fräse unter Vermeidung jedes Meißels umschnitten. Der aufgeklappte Knochen erwies sich auch an seiner Innenfläche gegenüber der äußeren Wunde intakt, ebenso die Dura. Incision derselben und Zurückklappen. Über der motorischen Region, etwa zwei Finger breit nach hinten von der Stelle der äußeren Wunde, ist die Arachnoidea in der Ausdehnung eines Fünfmarkstücks leicht getrübt. Die Sulci von Liquor stark angefüllt, die Gyri von ihm bedeckt. Gefäßinjektion lebhaft. Der Liquor sowie der Grund der Sulci rauchig getrübt. Liquor schwindet auf Druck nicht. Ritzen der Arachnoidea, wobei etwas Liquor abtropft; während dicht daneben die Quellung dieselbe bleibt, ein Auffangen ist nicht möglich. Das Gehirn erweist sich außer vermehrter Gefäßinjektion, stärkerer Schwellung nicht verändert, pulsiert gut. Diese Fünfmarkstück große Partie setzt sich deutlich durch die Vermehrung des Liquor, die entzündlichen Erscheinungen gegen die Umgebung ab. Einführen eines Tampons, Umkrempeln der Dura, Zurückklappen des Lappens. In den ersten 24 Stunden nehmen die Reizerscheinungen unter Ansteigen der Temperatur zu.

Nach 48 Stunden waren die paretischen und paralytischen Symptome gewichen. Die Wunde heilte wegen einer Knochennekrose nur langsam. Das Allgemeinbefinden und der übrige Zustand aber war vom zweiten Tage an ein guter. Benommenheit, die nach der Operation angedeutete Nackensteifigkeit, Lähmungserscheinungen in der linken Körperseite, alles war von der Operation an geschwunden und kehrte nicht wieder. Am II. IX., also ca. drei Monate nach der Operation, treten zwei Anfälle unvermittelt ein, die mit Zuckungen im linken Facialisgebiet begannen und auf den linken Arm und das linke Bein übergriffen und allmählich die gesamte Skelettmuskulatur befielen. Dauer etwa zwei Minuten. Am Schlusse des Anfalls länger dauernde extreme Stellung der Bulbi nach links unten. Sehachsen parallel. Kein Abgang von Urin und Kot, keine Aura, keine Amnesie. Patient empfindet, wie er nach dem Anfall klar angibt, die ersten Zuckungen im Gesicht sehr deutlich. Die Anfälle traten mit einem Intervall von ca. I5 Minuten auf; Patient fühlte sich etwas angegriffen. Bis zur Entlassung treten keine Anfälle mehr ein.

Entlassung sbef und am 6. X.: Erinnerungsvermögen gut. Linker Facialis funktioniert normal, kein Nystagmus. Pupillen reagieren prompt. Dynamometer links 65, rechts 50. Reflexe normal. Patellarreflex beiderseits etwas lebhaft, gleich, kein Fußklonus, kein 
Babinski, kein Romberg, keine Sensibilitätsprominenzen, keine Kopfschmerzen.

Epikrise: Da die progredienten Herderscheinungen das Krankheitsbild beherrschten - die Angaben über Krampfanfälle vor der Operation wurden erst später gemacht --, allgemeine Druckerscheinungen wenig ausgesprochen waren, Nackensteifig. keit nur angedeutet war, so sprach alles für einen Herd in der Hirnrinde, und zwar am Sitz der motorischen Zentren. Mit Rücksicht auf das vorhergegangene Trauma und die infizierte Weichteilwunde glaubte ich mit Bestimmtheit einen Kontusionsherd in der Nähe der Zentren, der nachträglich infiziert war und durch die Beteiligung der Umgebung - Ödem --- die motorischen Zentren ergriffen hatte, annehmen zu müssen. Ich hatte mir das Ganze als eine progrediente Encephalitis nach Trauma vorgestellt. Alles stimmte mit dieser Überlegung überein. Da der Knochen intakt war, mußte die Infektion durch den Knochen hindurch bis zum Kontusionsherde vordringen. Hierzu brauchte es Zeit. Das Intervall von 14 Tagen bis 3 Wochen sprach nicht dagegen. Anfänglich mußte der Kontusionsherd die motorischen Zentren unberührt gelassen haben und erst durch die spätere Infektion sie geschädigt haben. Hiermit stimmt der etwas nach vorn gelegene Sitz der äußeren Wunde und der Umstand überein, daß nicht eine vollständige Lähmung der kontralateralen Seite erfolgt und der Facialis am wenigsten beteiligt war. Demgegenüber war eine umschriebene Meningitis mit so ausgesprochenen, alle anderen Symptome bei weitem überragenden Herderscheinungen eine sehr große Seltenheit. Hätte ich mich mit einer weniger exakten Diagnose begnügt, nur einen die motorischen Zentren schädigenden infektiösen Prozeß angenommen, so wäre die Diagnose zwar weniger schön, aber richtig gewesen. Eins aber mußte als vorhanden mit aller Bestimmtheit angenommen werden und es hat sich auch bewahrheitet: $d$. i. ein infektiöser fortschreitender Prozeß. Ein Trauma, welches die motorischen Gebiete sekundär ergriffen hätte - müßte mit seinen reaktiven Prozessen früher einsetzen. Nur ein Neues konnte nach 3 Wochen so ausgesprochen fortschreitende Erscheinungen machen, und das konnte nur eine Infektion sein. Die eigentlichen Spätblutungen Bollingers sitzen an anderer Stelle, machen viel heftigere Erschei- 
nungen; für kleinere Blutungen in einem alten Kontusionsherd waren die allgemeinen Druckerscheinungen zu gering, die $\mathrm{Zu}$ nahme der Erscheinungen zu langsam. Auch der seit 8 Tagen bestehende Kopfschmerz ließ sich damit nicht erklären. Er deutet doch das Übergreifen des entzündlichen Prozesses auf die Meningen an. Es blieb also nur die eine oben erwähnte Annahme übrig. Diese gab die unbedingte Indikation zum operativen Vorgehen. Lag, wie angenommen, eine Encephalitis vor, so konnte nur durch Entfernung des infizierten progredienten Herdes die Zerstörung weiterer Hirnteile verhindert, der ev. letale Ausgang durch Durchbruch in den Ventrikel oder andere Komplikationen vermieden werden; lag eine Meningitis vor, so war es nur durch schnelles Handeln möglich, sie zu begrenzen. Wenn es auch sicher ist, daß diese Meningitiden ohne. Operation zum Stillstand kommen können - wie oft dieselben in die chronische Form übergehen, zur Cystenbildung führen und dann nachträglich noch die Operation indizieren, lasse ich dahingestellt - , so konnte ich mit dieser Eventualität angesichts der Zunahme der Erscheinungen im Interesse der Kranken nicht rechnen. Ich hatte es mit einer innerhalb des Schädels fortschreitenden, aber bis dahin auch im wesentlichen begrenzten Affektion zu tun, und ich glaube nicht, daß ein anderer Chirurg bei dieser Sachlage abgewartet hätte. Die Verantwortung schien mir zu groß.

Eine Lumbalpunktion angesichts der ausgesprochenen Herderscheinungen vorzunehmen, erschien mir therapeutisch nicht richtig, konnte unter Umständen schaden. War es ein encephalitischer Herd, so konnte sie nur durch Änderung der Spannung ev. entlastend auf die Umgebung des Herdes wirken. Da aber die allgemeinen Druckerscheinungen nur gering waren, so erschien mir diese Hoffnung nicht sehr groß und nicht sehr begründet; lag eine umschriebene Meningitis vor, so bildeten die intrameningealen Verwachsungen ein Hindernis für den Erfolg. Angesichts der deutlichen Herderscheinungen habe ich auf die Anwendung der Lumbalpunktion verzichtet und freue mich noch nachträglich, daß ich damit keine Zeit verloren habe. Von ev. nachteiligen Folgen will ich nicht sprechen. Hätten die allgeme in e n Druckerscheinungen in stärkerem Maße bestanden, dann war die Frage eine andere, dann konnte man die Lumbalpunktion 
als aussichtsvoll ansehen, dann hätte auch die Frage, ob allgemeine oder verbreitete Meningitis oder größerer encephalitischer Herd therapeutisch eine größere Wichtigkeit gehabt und wäre durch die Lumbalpunktion geklärt worden. Der eng umschriebene, infektiöse, auf die motorischen Zentren übergreifende Herd bedurfte einer weiteren Klärung nicht, und auch in diagnostischer Hinsicht hätte uns die Lumbalpunktion nicht weiter gebracht, selbst wenn sie etwas mehr Liquor unter höherem Druck ergeben hätte. Der Befund bei der Operation zeigte das deutliche Bild einer umschriebenen akuten Meningoencephalitis. Der Liquor leicht getrübt, vermehrt, die weichen Hirnhäute in umschriebener 5-Markstück großer Stelle deutlich entzündet, das darunterliegende Gehirn stärker gerötet, von verminderter Konsistenz, aber deutlich pulsierend. Beim Ritzen der Arachnoidea floß nur etwas Liquor $a b$, dicht daneben blieb die vermehrte Liquoransammlung bestehen; ein Zeichen, daß die Kommunikation durch Verwachsungen aufgehoben war. Die erkrankte Partie hob sich durch ihren Liquorreichtum, ihr Aussehen deutlich von den angrenzenden gesunden Bezirken ab. Das Aussehen der Gehirnflüssigkeit war leicht getrübt, rauchig, in den gesunden Partien klar. Dieses Aussehen spricht ja nicht unbedingt für die bakterielle Infektion, eine bakteriologische Untersuchung ist wegen Mangel an Liquormaterial unterblieben, aber trotzdem gibt es keine andere Möglichkeit als mit Rücksicht auf die eiternde äußere Wunde anzunehmen, daß die Infektion von hier aus durch Weiterwandern erfolgt ist und daß es sich um eine infektiöse Meningitis handelte. Auffallend, aber nicht dagegen sprechend ist, daß weder in der Dura noch im Knochen eine entzündete oder eitrige Stelle gefunden wurde. Es ist aber sehr wohl möglich, $\mathrm{da} B$ sie bestanden hat, sehr klein war, übersehen wurde. Es ist andererseits denkbar, daß sie schon so gut wie abgeheilt war. In dem Falle Friedrichs, wo die Messerklinge durch die Dura gedrungen war und unter ihr eine Encephalitis erzeugt hatte, waren auch entzündliche Veränderungen in der harten Hirnhaut nicht nachweisbar.

Da der erhaltene Operationsbefund völlig zur Erklärung genügte, das bloßliegende Gehirn deutliche Pulsation zeigte, so war ein weiteres Vorgehen nicht am Platze. Zur etwaigen Ab- 
leitung des Liquor, zur Druckregulierung krempelte ich die Dura um und legte einen kleinen lockeren Tampon auf die Stelle.

Der vorliegende Fall hat ein prinzipielles Interesse. Er zeigt uns eine akute infektiöse Meningoencephalitis in allerfrühstem Stadium mit ausgesprochenen progredienten, das ganze Krankheitsbild beherrschenden Herdsymptomen, die durch Trepanation geheilt ist und beweist die Berechtigung bzw. Notwendigkeit frühzeitiger Trepanation in diesen und ähnlichen Fällen.

Axhausen hält seinen Fall für traumatischer Natur, und wenn, wie ich oben hervorhob, gewisse Momente dagegen zu sprechen scheinen, so muß es doch auffallen, daß seine Annahme mit den Ergebnissen der Literatur, nach der traumatische, umschriebene Meningitiden früher in die Erscheinung traten, als auf Infektion oder anderer Basis beruhende, übereinstimmt. Bei Axhausen setzten die meningitischen Erscheinungen wenige Tage nach dem Trauma ein, bei Wendel vergingen 3 Monate, ehe nach der infektiösen Liderkrankung die seröse Meningitis sich einstellte. Das entspricht eigentlich gar nicht unseren sonstigen Erfahrungen, nach denen die Meningen so schnell und prompt auf jede Infektion reagieren. Die Sachlage ist jedoch eine wesentlich andere, wenn durch eine Verletzung eine direkte Freilegung und Infektion der Gehirnhaut erfolgt. Beim Trauma ohne Infektion und nachfolgender akuter umschriebener Meningitis trifft der Reiz eine umschriebene Stelle, verändert diese gegen die Umgebung mit einem Schlage und in der Peripherie setzen die reaktiven Prozesse ein, schließen die verletzte Partie gegen die Umgebung ab. So kann es geschehen, daß mit dem Einsetzen dieser Reaktion in den ersten Tagen schon eine umschriebene Partie der Hirnhaut durch Verklebungen und Verwachsungen abgeschlossen wird und damit der Grund zu einer umschriebenen Meningitis gelegt ist. In Wirklichkeit sind aber die akuten Formen weitaus seltener traumatischen Ursprungs als die chronischen, weil die Gewalteinwirkung keine zu große und auf das Gehirn tief übergreifende sein darf. Die harte Hirnhaut darf nicht verletzt, die weichen Hirnhäute dürfen nicht zerrissen sein. Bei den chronischen Formen braucht es dieser Einschränkung nicht; da können auch die Rinde mitschädigende. Traumen nachher zu dem Bilde einer umschriebenen Meningitis führen. Was anders 
ist es bei der Infektion oder der Nähe eines infizierten Herdes. Sind die Bakterien sehr virulent, so fehlt die Zeit zur Begrenzung durch intrameningeale Verwachsungen. Bei mäßig starker Infektion, bei dem allmählichen Vordringen des Infektionsherdes gegen die Meningen aber haben diese Zeit, sich lokal abzuschließen, ohne die Fähigkeit, Liquor zu produzieren, einzubüßen.

Die von den Otologen gemachten Beobachtungen dürfen nicht unberücksichtigt bleiben, in denen in der Nähe eitriger, infektiöser Herde sich eine Meningitis serosa circumscripta gebildet hat. Hier kann der Vorgang nur so erklärt werden, daß der nahe Infektionsherd entzündliche, intrameningeale Verwachsungen gezeitigt hat, innerhalb welcher dann die Exsudation des Liquor so lange erfolgte, bis äußere Symptome zur Eröffnung zwangen.

Die Resorptionsvorgänge scheinen bei Trauma unter Umständen viel stärker leiden zu können wie bei den von einem Infektionsherde fortgeleiteten Entzündungen. Bei letzteren, selbst wenn sie ausgesprochen eitrig sind - W endel-, braucht doch die Meningitis nicht diese Form anzunehmen. Wenn auch in meinem Falle der Liquor eine rauchige Trübung zeigte, ein eitriger Infektionsherd in der Nähe lag, so kann ich auch hier nicht eine vollvirulente, sondern nur eine abgeschwächte Infektion annehmen. Gewiß können auch volleitrige Meningitiden sich begrenzen, ihr Verlauf ist aber ein wesentlich stürmischerer und auch klinisch anderer als die uns beschäftigende zirkumskripte, akute, auf Infektion beruhende Meningitis.

In diesem frühen Stadium, wie ich es beobachten konnte, zeigte sich ferner die Beteiligung der Hirnrinde in ausgesprochener Weise. Einmal wiesen klinisch die Krampfanfälle, die ausgesprochenen Herderscheinungen darauf hin und dann wurde durch die Operation das pathologische Bild einer Entzündung der Hirnrinde einwandfrei erwiesen. Wie weit die Veränderungen in die Tiefe gereicht haben, vermag ich nicht zu sagen.

Und schließlich legt uns der Befund den Gedanken nahe, der auch von anderer Seite schon ausgesprochen ist, daß die Trübung des Liquor mit der Zeit schwinden und einem völlig klaren Aussehen Platz machen kann. Da es eine aseptische, eitrige Meningitis gibt, wie klinisch und experimentell erwiesen, so braucht diese Änderung nicht auf einem Absterben der Bakterien, womit 
ja auch zu rechnen sein wird, beruhen, sondern nur auf einem Schwinden der korpuskulären Beimengungen des Liquor.

Mit diesen Erörterungen haben wir uns der Genese der zirkumskripten chronischen Meningitis serosa immer mehr genähert. Es würde die mir gegebene Aufgabe zu weit überschreiten, wenn ich auf alle diesbezüglichen, namentlich in der letzten Zeit zahlreichen Beobachtungen eingehen wollte. Ich verweise da nur auf die Arbeiten Krauses, Borchardts, Wendels, Perthes und vor allen Dingen Zesas'. In seiner Zusammenstellung betrafen 9 das Kleinhirn, I I das Großhirn. Bei 6 sind Traumen, mehrfach Infektionskrankheiten, besonders Syphilis, vorhergegangen. Im wesentlichen kommen entzündungserregende Momente in Betracht. Die pathologisch-anatomischen Vorgänge bestehen in Verwachsungen der arachnoidealen Räume. Ihrer Genese, der ev. Beteiligung der Rinde habe ich wiederholt schon oben gedacht. Diagnostisch bereitet die Unterscheidung von Hirngeschwülsten große Schwierigkeiten. Denn die Remissionen und Intermissionen, welche vielfach die Meningitis serosa circumscripta auszeichnen, ich erinnere nur an den sehr interessanten Fall von Placzek und Oppenheim, kommen auch bei den Tumoren vor. Wenn auch die Infektion eine sehr häufige Ursache ist, so ist doch das Exsudat der serösen Meningitis meist steril. Auf dem Chirurgenkongresse 1912 teilte ich in der Diskussion zu dem Falle Wendels die Beobachtung einer Meningitis serosa circumscripta kurz mit. Da dieselbe anderweitig nicht veröffentlicht ist und einzelne Eigenheiten bietet, möchte ich den Fall hier kurz noch anführen:

43jähriger Mann, aufgenommen II. III. I9IO, entlassen am 6. V. I9Io. Seit ca. sechs Wochen Kopfschmerzen, die anfallsweise sehr heftig werden, dabei Erbrechen, worauf Erleichterung eintritt. Seit einer Woche täglich Erbrechen. Die Kopfschmerzen beginnen meist nachmittags und dauern bis Mitternacht. Vor 25 Jahren Hufschlag gegen die Stirn. Eine Stelle in der linken Stirngegend wird spontan und bei Beklopfen als besonders druckempfindlich bezeichnet.

F a milie na n a m nese: Frau war fünfmal gravide. Das erste Kind wurde mit Nacken- und Kreuzmeningocele geboren, das zweite Mal früher Abort, das dritte Mal lebendes Kind, I3 Jahre jetzt alt, epileptisch und geistesschwach, das vierte Mal einen Monat zu früh geboren, tot. Das fünfte Kind lebt und ist gesund, drei Jahre alt. Angeblich während einer Augenaffektion vor sechs Jahren auch An- 
fälle von Kopfschmerzen mit Erbrechen, die dann sistierten. Luetische Infektion nicht bekannt.

B e f u n d: Kräftiger Mann, etwas deprimiertes Wesen. Während der Kopfschmerzen häufig Kribbeln in der linken Hand. Augenhintergrund normal. Schädel normal. Links deutliche Cubitaldrüse. Trigeminus nirgends druckempfindlich. An den Hirnnerven nichts Besonderes. Motilität und Reflexe an den Extremitäten normal. Temperatur normal. Puls besonders während der starken Kopfschmerzen deutlich verlangsamt.

Bei diesen wenig ausgeprägten Erscheinungen konnte nur an einen raumbeengenden Proze $B$ in der linken Stirngegend gedacht werden. Da auf Jodkali keine Besserung erfolgte, inzwischen leichte Temperatursteigerungen unter Frost $\mathrm{ab}$ und $\mathrm{zu}$ eingetreten waren, die Schmerzhaftigkeit immer auf eine bestimmte Stelle in der Gegend des linken Stirnhirns hinwies, wurde in der Annahme eines Tumors oder eines sonstigen raumbeengenden Prozesses an dieser Stelle cingegangen.

Die Operation am 6. IV. legte nach Umstechung der Art. temp. und auricular. poster. osteoplastisch ohne nennenswerte Blutung das linke Stirnhirn frei. Die Schädeldecke ist außerordentlich stark verdickt und sklerotisch, die Dura pulsiert nur wenig. Nach Incision derselben sprudelt klare Gehirnflüssigkeit unter starkem Druck hervor. Die Pia ist stark injiziert, die Gefäße erscheinen wie in sulzige Massen eingebettet. Naht der Dura. Zwischen Knochenlappen und Dura wurde ein Tampon eingelegt. Heilung. Patient wurde beschwerdefrei entlassen. Auch nach der vor einiger Zeit erfolgten Nachuntersuchung ist Patient vollkommen beschwerdefrei und arbeitsfähig.

Es handelt sich hier also um einen typischen Fall von Meningitis serosa circumscripta chronica mit ausgesprochenen Remissionen und Intermissionen, der durch die Operation einer völligen und dauernden Heilung zugeführt wurde. Da die Erkrankung am Stirnhirn saß, so konnten Herderscheinungen nicht zum Ausbruch kommen und für die sonst typischen Erscheinungen des Stirnhirns war die Alteration dieses Hirnteiles nicht stark genug. Ätiologisch ist von Bedeutung, daß Trauma und Lues in Frage kamen. Wenn auch der Patient selbst sich keiner Infektion bewußt war, so deutete doch die Familienanamnese mit aller Entschiedenheit darauf hin. Zu dieser luetischen Disposition trat nun ein Trauma, das allerdings 25 Jahre zurücklag, aber doch die Gegend der späteren Erkrankung betroffen hatte. Wenn man bedenkt, wie langsam und spät oft posttraumatische Meningitiden in die Erscheinung treten, und daß in unserem Falle die 
ersten Anfänge des Leidens mindestens 6 Jahre vorher sich bemerkbar machten, so wird man die Bedeutung des Traumas nicht außer acht lassen können. Luetische Späterkrankungen siedeln sich aber gern auf dem Boden eines Traumas an, und so liegt es nahe, auch in unserem Falle ein Zusammenwirken beider Momente anzunehmen. Gegen einen Tumor sprach diagnostisch, daß Verschlimmerungen des Leidens einer über Jahre dauernden Periode vollkommenen Wohlbefindens gefolgt waren; da aber alle Erscheinungen so wenig ausgesprochen waren, konnte eine Meningitis serosa nur vermutet werden; es konnte auch eine umschriebene Pachymeningitis oder ähnliches vorliegen.

Therapeutisch bin ich so vorgegangen, daß ich zwar die Dura mit einigen Stichen nähte, daß ich aber darauf bedacht war, sie vollkommen in das Bett der Flüssigkeitsansammlung einschlagen zu können. Die Heilung dieser zirkumskripten Meningitiden unter dem Einfluß der Operation haben wir uns so vorzustellen, daß die Dura mit dem Gehirn und den Häuten verwächst, so daß einmal ein Hohlraum oder Maschen zwischen beiden nicht mehr sich bilden können und zweitens durch diese Verwachsungen Exsudation und Resorption sowie die Zirkulation geändert wird. Ich habe auf diese Dinge schon in der Diskussion auf dem Chirurgenkongreß hingewiesen. Da bei allen bisherigen Operationen die Dura gespalten wurde, so war damit schon ein wichtiger Schritt zur Heilung getan; wo es aber unterlassen war, die intrameningealen Hohlräume zu entleeren, die Verwachsung zu zerreißen, da konnte sich die Dura nicht fest genug anlegen, da kamen Rezidive zustande. Diese Vorgänge geben auch eine Erklärung dafür, daß nach schweren Gehirn- und Schädelverletzungen mit Zerstörung des Gehirns und Durchreißung der Hirnhäute keine Meningitis serosa - soweit mir bekannt - beobachtet ist. Nur die leichteren Schädigungen, die wohl die Hirnhaut reizen, aber nicht durchreißen, geben Veranlassung zur Entstehung der Meningitis circumscripta. Das ist auch anamnestisch von Bedeutung.

Trotzdem in unserem Falle Lues im Spiel war, zeigte sich Jodkali erfolglos, was nicht weiter wunderbar ist. In dem Falle Placzeks und Borchardts hatte Jodkali zweimal Erfolg, bis es zum dritten Mal versagte. Die Wirkung ist also selbst bei 
syphilitischer Ätiologie eine unzuverlässige, und da die zirkumskripten Meningitiden bei Operation eine so günstige Prognose bieten, so ist es nicht zweckmäßig, sich lange bei so zweifelhaften internen Versuchen aufzuhalten.

Es kam mir in der vorliegenden Arbeit vor allen Dingen darauf an, die Aufmerksamkeit auf das Krankheitsbild der akuten progredienten Encephalitis, Meningitis circumscripta acuta und Meningoencephalitis zu lenken, darzutun, daß die letzteren beiden Krankheitsformen genau unter demselben Bilde wie die Encephalitis mit ausgesprochenen progredienten Herdsymptomen auf infektiöser Basis entstanden, verlaufen können und durch die Zunahme der Erscheinungen, die Gefahr der fortschreitenden Infektion die sofortige Operation, d.h. die Trepanation fordern und sehr günstige Aussichten auf Heilung bieten. Die klinische Trennung der drei Krankheitsformen wird nur selten möglich sein und wir tun deshalb besser, sie unter denselben Gesichtspunkten, in demselben Kapitel zu betrachten.

Außerdem liefern uns diese akuten Formen wertvolle Beiträge für die Genese der Meningitis circumscripta chronica und bringen den Beweis, daß auch infektiöse Liquoransammlungen von trüber Beschaffenheit in späterer Zeit als einfache klare Cysten usw. erscheinen können.

\section{Literaturverzeichnis.}

I. Axha usen. Berliner klin. Wochenschr. 1909 .

2. Bendert u. Nieuwenhuyse. Münchn. med. Wochenschr. I9Io.

3. B ergma n n. Deutsche Chirurgie und Sammlung klin. Vorträge, Nr. Ior.

4. B orchard. Verhandlungen, Chirurgen-Kongreß IgI 2.

5. Borsuk u. Wizel. Arch. f. klin. Chir., 54.

6. Brewill. Arch. f. klin. Chir., Bd. 79.

7. Brugie r. Deutsche med. Wochenschr. I9r2.

8. Friedrich. Deutsche Zeitschr. f. Chir., Bd. 85 .

9. Grabs. Dissertat. Leipzig I9I I.

Io. Gradenigo. Arch. f. Ohrenheilkunde, Bd. 47.

I1. Gu $\mathrm{B}$ en ba u e r. Sitzungsbericht der Prager Ver. d. Ärzte, Io. II. $1 \delta 93$.

12. H a hn. Deutsche med. Wochenschr. 1896.

13. J a nz. Münchn. med. Wochenschr. I909.

14. Koch. Dissertat. Marburg 1909.

15. Krause, Chirurgie des Gehirns und Rückenmarks. 
I6. Kra use u. Placzek. Berliner klin. Wochenschr. 1907.

17. K ü mm el. Arch. f. klin. Chir., 77.

18. $\mathrm{K} \ddot{\mathrm{u}} \mathrm{t} \mathrm{t}$ e r. Handbuch der Chirurgie.

19. M a c Ew en, Die infektiöseitrigen Erkrankungen des Gehirns und Rückenmarks.

2o. M a rtin du Pau, Ch. Revue de la Suisse rom. I9Ix.

2r. Muskens. Münchn. med. Wochenschr. I9Io.

22. Oppenheim u. Borchardt. Deutsche med. Wochenschr. I9ro.

23. P e r thes. Verhandlungen, Chirurgen-KongreB I9I2.

24. Phelps. Hildebrands Jahresberichte, Bd. I5.

25. Pitterlein, Zur Kasuistik der Hirnchirurgie. Münchn. med. Wochenschrift 1909.

26. Pram bolini. Zentralbl. f. Chir. r9ir.

27. Quincke. Münchn. med. Wochenschr. I9Io.

28. Remling er. Deutsche med. Wochenschr. I9II.

29. R e u B. Dissertat. Tübingen r9I I.

3o. Rütlin. Münchn. med. Wochenschr. I9I2.

3I. S cha ef er. Inaug.-Diss. Bonn I9II.

32. Scha uch. Beitr. z. klin. Chir,, Bd. 69.

33. Schloffer. Beitr. z. klin. Chir., Bd. 22.

34. Schwarz. Deutsche Zeitschr. f. Chir, 124.

35. Streit. Deutsche med. Wochenschr. I912.

36. Uffenorde. Deutsche med. Wochenschr. I9I2.

37. Völs ch. Münchn. med. Wochenschr. I9I2.

38. W endel. Verhandlungen, Chirurgen-Kongre $B$ I9Iz.

39. Z a bel. Deutsche med. Wochenschr. I9I2.

40. Z e s a s. Sammlung klinischer Vorträge, Nr. 685 . 